\title{
NETWORVINAS AND SEGURVIV ISSUES
}

\section{Mohamed M. El Hadi}

\section{INTRODUCTION:}

Many organizations have invested vast amount of money in computer networks, only to find out that although it is providing means of improving the efficiency and productivity of the organization but it also exposes the Organization to possible attacks and threats. Such attacks have been the most challenging issue for most network administrators and a worrying topic for administrators.

Organizations need to share services resources and information but they still need to protect these from people who should not have access to them, while at the same time making those resources available to authorized users. Effective security achieves these goals.

The greatest threat to computer systems and their information comes from humans, through actions that are either malicious or ignorant. When the action is malicious, some motivation or goal is generally behind the attack. For instance, the goal could be to disrupt normal business operations, thereby denying data availability and production.

Most of the attacks are becoming more frequent and more damaging, and they are using well-known techniques and methods to exploit vulnerability in security policies and systems.

\section{Network System}

The most popular term - LAN or Local Area Network is a computer network (or data communications network) which is confined to a room, a building, or a group of adjacent buildings. A similar network on a larger scale is sometimes referred to as a WAN (Wide Area Network), or in some cases more specifically, a MAN (Metropolitan Area Network) if it is confined to a single metropolitan area.
The term LAN is most often used to refer to networks created out of a certain class of networking equipment which is tailored to communication over a short distance. This is in contrast to networks, which happen to span short distances, yet are constructed using "WAN" equipment (i.e., equipment capable of transmitting long distances). LAN-style networking equipment typically transmits data at a higher rate than WAN-style equipment: the equipment's design takes advantage of the short distance to supply a high transmission-rate at a relatively low cost.

Network access using a modem and ordinary telephone line, note that both LAN and WAN equipment typically offers faster data transfer than even the fastest ordinary modem/phone-line access, LAN transfers being on the order of a million times faster. This means graphics that are loaded through the network can be displayed significantly faster, and that there are things that it is practical to do on a LAN that you would never do with a modem: for example, you might set up your computer to load your word processing application through the LAN rather than from hard disk; the time you have to wait while it loads would be similar (a few seconds) in either case. In contrast, loading such an application through a modem would require minutes or hours.

A typical use of a LAN is to tie together personal computers in an office in such a way that they can all use a single printer and a file server (briefly, a file server is a computer set up so that other computers can access its hard disk as if it were their own). LANs are also used to transmit e-mail between personal computers in an office, or to attach all the personal computers in the office to a WAN or to the Internet.

There is some variation in the way the term LAN is used:

- It is used to refer to a file server and printer, and often the personal computers that are tied to them. People refer to saving their files on the LAN, or on the PC LAN.

- It is used more specifically to refer to the data communications wiring and equipment that ties the personal computers to the file server and the printer.

One of the terms associated with most Networks is Ethernet, the most common type in use today. Ethernet is an example of what is called a LAN technology, or in 
the more specific sense of the word LAN, one of several types of LANs. Some other types of LANs are Token Ring, FDDI, and Fast Ethernet.

\section{What is the Internet?}

The Internet is the world's largest network of networks. When you want to access the resources offered by the Internet, you don't really connect to the Internet; you connect to a network that is eventually connected to the Internet backbone, a network of extremely fast (and incredibly overloaded!) networks components. This is an important point: the Internet is a network of networks -- not a network of hosts.

\section{Defining Security}

Computer security is about protecting information. Lately it includes privacy, confidentiality, and integrity.

We need to know the value of the information as defined above in order to develop protective measures that will protect the information from the outside world, while allowing known individuals with unique identities the access required. Here are some protective measures to consider:

\section{Prevention:}

Take measures that prevent your information from being damaged, altered, or stolen. Preventive measures can range from locking the server room door to setting up high-level security policies.

\section{Detection:}

Take measures that allow you to detect when information has been damaged, altered, or stolen, how it has been damaged, altered, or stolen, and who has caused the damage. Various tools are available to help detect intrusions, damage or alterations, and viruses.

\section{Reaction:}

Take measures that allow recovery of information, even if information is lost or damaged.

The above measures are all very well, but if we do not understand how information may be compromised, we cannot take measures to protect it. Here are some components that we can examine on how information can be compromised:

\section{Confidentiality:}

The prevention of unauthorized disclosure of information. This can be the result of poor security measures or information leaks by personnel. An example of poor security measures would be to allow anonymous access to sensitive information.

\section{Integrity:}

The prevention of erroneous modification of information. Authorized users are probably the biggest cause of errors and omissions and the alteration of data. Storing incorrect data within the system can be as bad as losing data. Malicious attackers also can modify, delete, or corrupt information that is vital to the correct operation of business functions.

\section{Availability:}

The prevention of unauthorized withholding of information or resources. This does not apply just to personnel withholding information. Information should be as freely available as possible to authorized users.

\section{Authentication:}

The process of verifying that users are who they claim to be when logging onto a system. Generally, the use of user names and passwords accomplishes this. More sophisticated is the use of smart cards and retina scanning. The process of authentication does not grant the user access rights to resources - this is achieved through the authorization process.

\section{Authorization:}

The process of allowing only authorized users access to sensitive information. An authorization process uses the appropriate security authority to determine whether a user should have access to resources.

\section{The Need for Security}

Administrators normally find that putting together a security policy that restricts both users and attacks is time consuming and costly. Users also become disgruntled at the heavy security policies making their work difficult for no discernable reason, causing bad politics within the company. Planning an audit policy on huge networks takes up both server resources and time, and often administrators take no note of the audited events. A common attitude among users is that if no secret work is being performed, why bother implementing security.

There is a price to pay when a half-hearted security plan is put into action. It can result in unexpected disaster. A password policy that allows users to use blank or weak passwords is a hacker's paradise. No firewall or proxy protection between the organization's private local area network (LAN) and the public Internet makes the company a target for cyber crime.

Organizations will need to determine the price they are willing to pay in order to protect data and other assets. This cost must be weighed against the costs of losing information and hardware and disrupting services. 
The idea is to find the correct balance. If the data needs minimal protection and the loss of that data is not going to cost the company, then the cost of protecting that data will be less. If the data is sensitive and needs maximum protection, then the opposite is normally true.

\section{Security Threats Introduction}

The first part of this section outlines security threats and briefly describes the methods, tools, and techniques that intruders use to exploit vulnerabilities in systems to

\section{achieve their goals.}

Security Threats, Attacks, and Vulnerabilities

Information is the key asset in most organizations. Companies gain a competitive advantage by knowing how to use that information. The threat comes from others who would like to acquire the information or limit business opportunities by interfering with normal business processes.

The object of security is to protect valuable or sensitive organizational information while making it readily available. Attackers trying to harm a system or disrupt normal business operations exploit vulnerabilities by using various techniques, methods, and tools.

Attackers generally have motives or goals - for example, to disrupt normal business operations or steal information. To achieve these motives or goals, they use various methods, tools, and techniques to exploit vulnerabilities in a computer system or security policy and controls.

\section{Goal + Method + Vulnerabilities $=$ Attack}

\section{Security Threats}

Threats can originate from two primary sources: humans and nature. Human threats subsequently can be broken into two categories: malicious and non-malicious. The non-malicious "attacks" usually come from users and employees who are not trained on computers or are not aware of various computer security threats. Malicious attacks usually come from non-employees or disgruntled employees who have a specific goal or objective to achieve.

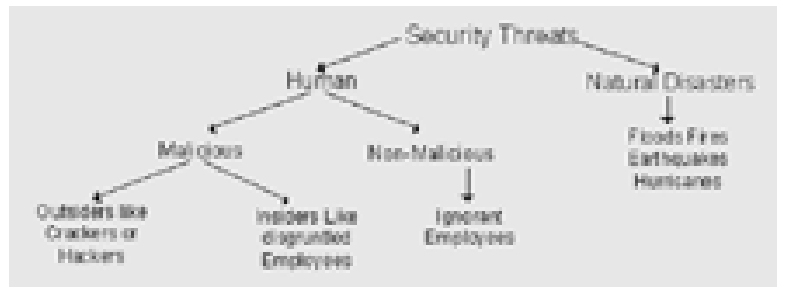

Figure 1 introduces a layout that can be used to break up security threats into different areas.

\section{Natural Disasters}

Nobody can stop nature from taking its course. Earthquakes, hurricanes, floods, lightning, and fire can cause severe damage to computer systems. Information can be lost, system downtime or loss of productivity can occur, and damage to hardware can disrupt other essential services.

Few safeguards can be implemented against natural disasters. The best approach is to have disaster recovery plans and contingency plans in place. Other threats such as riots, wars, and terrorist attacks could be included here. Although they are human-caused threats, they are classified as disastrous.

\section{Human Threats}

Malicious threats consist of inside attacks by disgruntled or malicious employees and outside attacks by nonemployees just looking to harm and disrupt an organization.

The most dangerous attackers are usually insiders (or former insiders), because they know many of the codes and security measures that are already in place. Insiders are likely to have specific goals and objectives, and have legitimate access to the system. Employees are the people most familiar with the organization's computers and applications, and they are most likely to know what actions might cause the most damage.

The insider attack can affect all components of computer security. By browsing through a system, confidential information could be revealed. Insider attacks can affect availability by overloading the system's processing or storage capacity, or by causing the system to crash.

People often refer to these individuals as "crackers" or "hackers." The definition of "hacker" has changed over the years. A hacker was once thought of as any individual who enjoyed getting the most out of the system he or she was using. A hacker would use a system extensively and study it until he or she became proficient in all its nuances. This individual was respected as a source of information for local computer users, someone referred to as a "guru" or "wizard."

However, the term hacker refers to people who either break in to systems for which they have no authorization or intentionally overstep their bounds on systems for which they do not have legitimate access.

The correct term to use for someone who breaks in to systems is a "cracker." Common methods for gaining access to a system include password cracking, exploiting known security weaknesses, network spoofing, and social engineering.

Malicious attackers normally will have a specific goal, objective, or motive for an attack on a system. These goals could be to disrupt services and the continuity of business operations by using denial-of-service (DoS) attack tools. They might also want to steal information 
or even steal hardware such as laptop computers. Hackers can sell information that can be useful to competitors.

In 1996, a laptop computer was stolen from an employee of Visa International that contained 314,000 credit card accounts. The total cost to Visa for just canceling the numbers and replacing the cards was \$6 million.

SecurTek Corporation, http://www.securtekcorporation.com/Protect1.ht

Attackers are not the only ones who can harm an organization. The primary threat to data integrity comes from authorized users who are not aware of the actions they are performing. Errors and omissions can cause valuable data to be lost, damaged, or altered.

Non-malicious threats usually come from employees who are untrained in computers and are unaware of security threats and vulnerabilities.

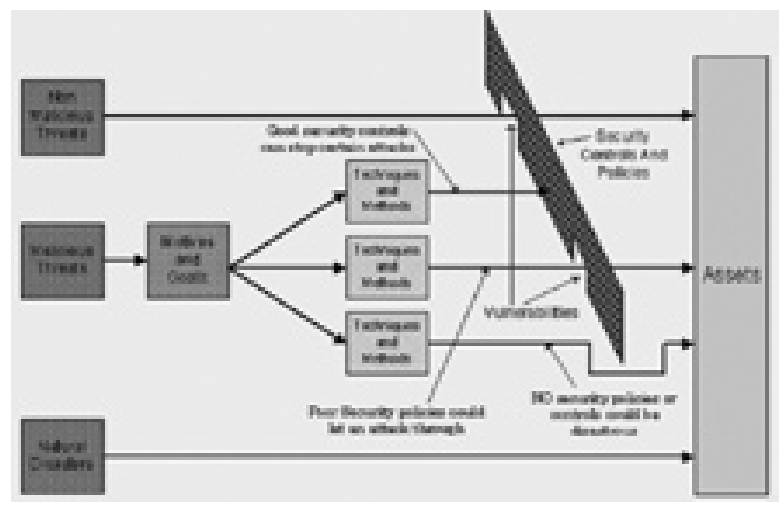

Figure 2 Non-Malicious Threats

The following table gives some examples of the various aspects discussed above.

Table 1: Various Threats and their Motives, Methods and Security Policies

\begin{tabular}{|c|c|c|c|}
\hline ICETS & 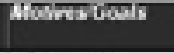 & Nistaxis & $\begin{array}{l}3 \text { secoury } \\
\text { Pelloches }\end{array}$ \\
\hline 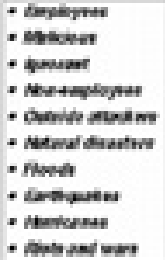 & 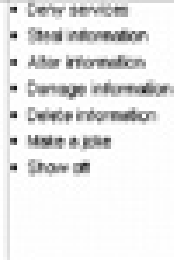 & 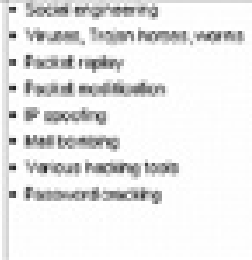 & 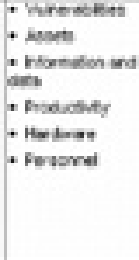 \\
\hline
\end{tabular}

Note that ignorant employees usually have no motives and goals for causing damage. The damage is accidental. Also, malicious attackers can deceive ignorant employees by using "social engineering" to gain entry. The attacker could masquerade as an administrator and ask for passwords and user names. Employees who are not well trained and are not security aware can fall for this. Common examples of computer-related employee sabotage include:
- Changing data

- Deleting data

- Destroying data or programs with logic bombs

- Crashing systems

- Holding data hostage

- Destroying hardware or facilities

- Entering data incorrectly

Motives, Goals, and Objectives of Malicious Attackers

There is a strong overlap between physical security and data privacy and integrity. Indeed, the goal of some attacks is not the physical destruction of the computer system but the penetration and removal or copying of sensitive information. Attackers want to achieve these goals either for personal satisfaction or for a reward.

Some methods that attackers use are as follows:

- Deleting and altering information. Malicious attackers who delete or alter information normally do this to prove a point or take revenge for something that has happened to them. Inside attackers normally do this to spite the organization because they are disgruntled about something. Outside attackers might want to do this to prove that they can get in to the system or for the fun of it.

- Committing information theft and fraud. Information technology is increasingly used to commit fraud and theft. Computer systems are exploited in numerous ways, both by automating traditional methods of fraud and by using new methods. Financial systems are not the only ones subject to fraud. Other targets are systems that control access to any resources, such as time and attendance systems, inventory systems, school grading systems, or long-distance telephone systems.

- Disrupting normal business operations. Attackers may want to disrupt normal business operations. In any circumstance like this, the attacker has a specific goal to achieve. Attackers use various methods for denial-ofservice attacks; the section on methods, tools, and techniques will discuss these.

Methods, Tools, and Techniques for Attacks

Attacks $=$ motive + method + vulnerability. The method in this formula exploits the organization's vulnerability in order to launch an attack as shown in Figure 2. Malicious attackers can gain access or deny services in numerous ways. Here are some of them:

- Viruses. Attackers can develop harmful code known as viruses. Using hacking techniques, they can break into systems and plant viruses. Viruses in general are a threat to any environment. They come in different forms and although not always malicious, they always take up time. Viruses can also be spread via e-mail and disks.

- Trojan horses. These are malicious programs or software code hidden inside what looks like a normal pro- 
gram. When a user runs the normal program, the hidden code runs as well. It can then start deleting files and causing other damage to the computer. Trojan horses are normally spread by e-mail attachments. The Melissa virus that caused denial-of-service attacks throughout the world in 1999 was a type of Trojan horse.

- Worms. These are programs that run independently and travel from computer to computer across network connections. Worms may have portions of themselves running on many different computers. Worms do not change other programs, although they may carry other code that does.

- Password cracking. This is a technique attackers use to surreptitiously gain system access through another user's account. This is possible because users often select weak passwords. The two major problems with passwords is when they are easy to guess based on knowledge of the user (for example, wife's maiden name) and when they are susceptible to dictionary attacks (that is, using a dictionary as the source of guesses).

- Denial-of-service attacks. This attack exploits the need to have a service available. It is a growing trend on the Internet because Web sites in general are open doors ready for abuse. People can easily flood the Web server with communication in order to keep it busy. Therefore, companies connected to the Internet should prepare for (DoS) attacks. They also are difficult to trace and allow other types of attacks to be subdued.

- E-mail hacking. Electronic mail is one of the most popular features of the Internet. With access to Internet e-mail, someone can potentially correspond with any one of millions of people worldwide. Some of the threats associated with e-mail are:

Impersonation. The sender address on Internet e-mail cannot be trusted because the sender can create a false return address. Someone could have modified the header in transit, or the sender could have connected directly to the Simple Mail Transfer Protocol (SMTP - the protocol used for sending e-mail) port on the target computer to enter the e-mail.

Eavesdropping. E-mail headers and contents are transmitted in the clear text if no encryption is used. As a result, the contents of a message can be read or altered in transit. The header can be modified to hide or change the sender, or to redirect the message.

- Eavesdropping. This allows a cracker (hacker) to make a complete copy of network activity. As a result, a cracker can obtain sensitive information such as passwords, data, and procedures for performing functions. It is possible for a cracker to eavesdrop by wiretapping, using radio, or using auxiliary ports on terminals. It is also possible to eavesdrop using software that monitors packets sent over the network. In most cases, it is difficult to detect eavesdropping.

- Social engineering. This is a common form of crack- ing. It can be used by outsiders and by people within an organization. Social engineering is a hacker term for tricking people into revealing their password or some form of security information.

- Intrusion attacks. In these attacks, a hacker uses various hacking tools to gain access to systems. These can range from password-cracking tools to protocol hacking and manipulation tools. Intrusion detection tools often can help to detect changes and variants that take place within systems and networks.

\section{Security Vulnerabilities}

As explained previously, a malicious attacker uses a method to exploit vulnerabilities in order to achieve a goal. Vulnerabilities are weak points or loopholes in security that an attacker exploits in order to gain access to the network or to resources on the network (see Figure 2). Remember that the vulnerability is not the attack, but rather the weak point that is exploited. Here are some of the weak points:

- Passwords. Password selection will be a contentious point as long as users have to select one. The problem usually is remembering the correct password from among the multitude of passwords a user needs to remember. Users end up selecting commonly used passwords because they are easy to remember. Anything from birthdays to the names of loved ones. This is vulnerability because it gives others a good chance to guess the correct password.

- Protocol design. Communication protocols sometimes have weak points. Attackers use these to gain information and eventually gain access to systems.

- Modems. Modems have become standard features on many desktop computers. Any unauthorized modem is a serious security concern. People use them not just to connect to the Internet, but also to connect to their office so they can work from home. The problem is that a modem is a means of bypassing the "firewall" that protects a network from outside intruders. A hacker using a "war dialer" tool to identify the modem telephone number and a "password cracker" tool to break a weak password can gain access to the system. Due to the nature of computer networking, once a hacker connects to that one computer, the hacker can often connect to any other computer in the network.

Some Examples of Security Threats

Example 1: Non-Malicious Threat (ignorant employees).

An employee known here as John Doe copies games and other executables from a 1.44 MB disk onto his

local hard drive and then runs the executables. Unfortunately, the games contained various viruses and

Trojan horses. The organization had not yet deployed any anti-virus software. After a short time, John Doe 
and other employees began to notice strange and unforeseen events occurring on their computers, causing disruption of services and possible corruption of data. The following figure explains the various vulnerabilities that existed and the loss in assets that are involved.

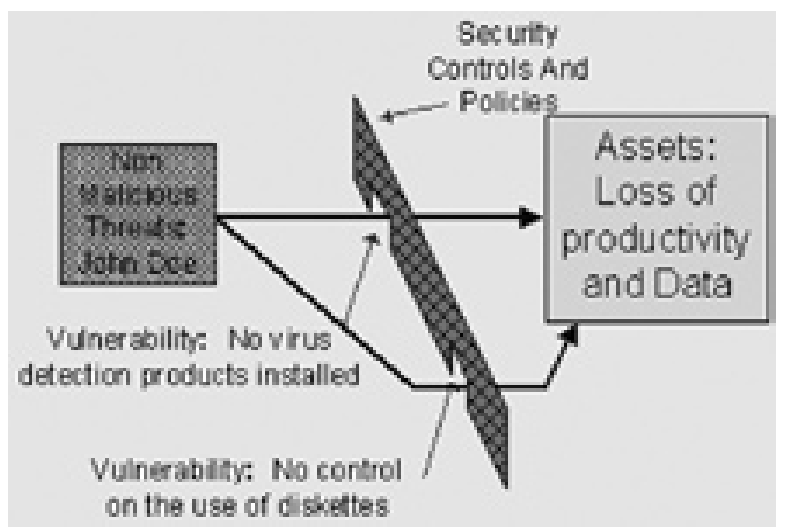

Figure 3 Non-Malicious Threat

Example 2: Malicious Threat (malicious attackers) An employee known here as Sally was turned down for promotion three times. Sally believes that she has put in a considerable amount of work and overtime and is being turned down for promotion because she is too young. Sally has a degree in computer science and decides to resign from the company and take

revenge on it by causing the company's Web server to stop servicing requests. Sally uses a denial-of

service attack tool called Trin00 to start an attack on the company's Web server.

Most of the company's business is conducted via ecommerce and clients are complaining that they cannot connect to the Web server. The following diagram outlines the various tools and vulnerabilities Sally used to achieve her goal.

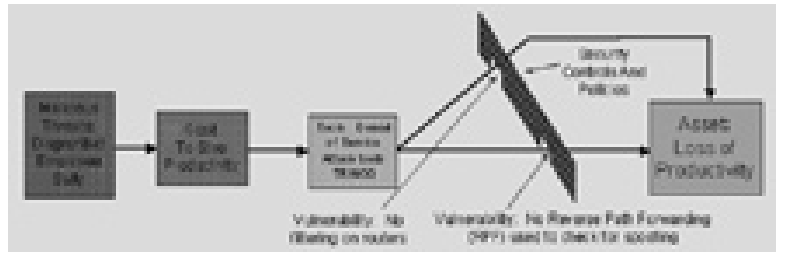

Figure 4 Malicious Threat (malicious attackers)

Example 3: Natural Disasters

An organization has various modems and Integrated Services Digital Network (ISDN) router installations and does not have surge protection. During a thunderstorm, lightning strikes the telephone and ISDN lines. All modems and ISDN routers are destroyed, taking with them a couple of motherboards. The fol- lowing diagram shows the vulnerability and the loss of assets.

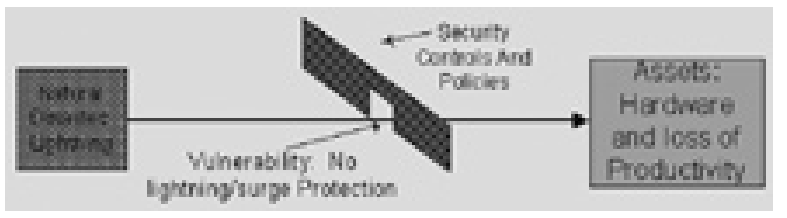

Figure 5 Natural Disasters

\section{Security Policies and plans}

Security Policies are the foundation, the bottom line of information security of an organization. Each organization would present a different policy plan that is appropriate, clear and effective for the organization.

\section{Design and implement a security plan.}

Designing a security plan includes setting security goals and strategies and deciding on the level of security that is appropriate. Deciding on the level of security means weighing the pros and cons of higher versus lower security. Higher security requires more administration but ensures only the right people will have access to your resources. Lower security creates a more flexible and open environment, but might not be as secure as other configurations.

\section{Understand and implement security policy.}

Security policy enforces uniform security standards for groups of users. Security policy is used to establish a basis of security for the environment. Different from user rights and permissions, security policy applies to all users or objects in the deployment.

\section{Planning for Security.}

Although security technologies are highly advanced, effective security must combine technology with good planning for business and social practices. No matter how advanced and well implemented the technology is, it is only as good as the methods used in employing and managing it.

Implementing the appropriate security standards is a key issue for most organizations. To implement security standards, devise a security plan that applies a set of security technologies consistently to protect the organization's resources.

A typical security plan might include the following sections:

- Security goals: Describe what the organization needs protecting.

- Security risks: Enumerate the types of security hazards that affect the enterprise, including what poses the threats and how significant the threats are. 
- Security strategies: A description of the general security strategies necessary to meet the threats and mitigate the risks.

- Security group descriptions: Describe security groups and their relationship to one another. This section maps security policies to security groups.

- Security Policy: Describe Group Policy security settings, such as network password policies.

- Network logon and authentication strategies: In a networked environment, consider authentication strategies for logging on to the network and for using remote access or smart card to $\log$ on.

- Information security strategies: How to implement information security solutions, such as an encrypted file system (EFS), Internet Protocol security, and access authorization using permissions.

- Administrative policies: Include policies for delegation of administrative tasks and monitoring of audit logs to detect suspicious activity.

For starters, the easiest way to deal with security policies is to use some pre-written "off the shelf". This is certainly a reasonable approach, but it is important to ensure that the policies are of the requisite standard, and perhaps are compliant with standards.

\section{An example:}

http://www.securitypolicy.co.uk/secpolicy/

\section{Conclusion}

Malicious attackers will use various methods, tools, and techniques to exploit vulnerabilities in security policies and controls to achieve a goal or objective. Non-malicious attacks occur due to poor security policies and controls that allow vulnerabilities and errors to take place. Natural disasters can occur at any time, so organizations should implement measures to try to prevent the damage they can cause.

\section{Reference:}

1. Bagwill, Robert, and Barbara Guttman. Internet Security Policy: A Technical Guide. National Institute of Standards and Technology Computer Security Division.

\section{http://csrc.nist.gov/isptg/html/}

2. Bassham, Lawrence E., and W. Timothy Polk. Threat Assessment of Malicious Code and Human Threats. National Institute of Standards and Technology Computer Security Division.

http://csrc.nist.gov/nistir/threats/

3. Bort, Julie. A False Sense of Security. Lantimes. http://www.lantimes.com/98/98jul/807b023a.html

4. Brown, Carol E. and Alan Sangster. Electronic Sabotage.

http://www.bus.orst.edu/faculty/brownc/lectures/virus/virus.htm

5. Chess, David. Things that Go Bump in the Net. http://www.research.ibm.com/massive/bump.html/

6. Huegen, Craig. Network-Based Denial of Service Attack Information.

http://users.quadrunner.com/chuegen/smurf/

7. Martin, Brian. Have Script Will Destroy (Lessons in DoS).

http://www.attrition.org/

8. Null, Christopher. Is the Hacker Threat Real? Lantimes.

http://www.lantimes.com/98/98mar/803b007a.html

9. Parker, Donn. Automated Crime.

http://www.infosecuritymag.com/

10. DDOS Debriefing.

http://www.infosecuritymag.com/

11. Department of Defense Trusted Computer System Evaluation Criteria (Orange Book). National 12

12. Computer Security Center.

http://csrc.ncsl.nist.gov/secpubs/rainbow/std001.txt

12 Trusted Network Interpretation (Red Book). National Computer Security Center.

http://csrc.ncsl.nist.gov/secpubs/rainbow/tg005.txt

\section{Web Sites}

For more information on viruses, Trojan horses, and Internet hoaxes, see:

- The Computer Incident Advisory Capability site at http://ciac.llnl.gov

- The E-Commerce Webopedia at http://e-comm.webopedia.com/

- http://www.cert.org

- http://www.blueroom.com/internet/

For more information on distributed denial-of-service attacks, see http://www.icsa.net/

For more information on back-end system issues for online financial sites, see http://www.incurrent.com/ For more information about security, see the Pretty Good Privacy site at http://www.pgp.com.

Additional sites on security issues:

http://www.nwfusion.com/

http://www.nai.com/

http://www.cert.org/

http://www.antionline.com/

http://www.infosyssec.com/ 